\title{
Bioprocess development for 2,3-butanediol production from crude glycerol and conceptual process design for aqueous conversion into methyl ethyl ketone
}

Sofia Maina ${ }^{a, \hat{t}}$, Endrit Dheskali ${ }^{b, c, \hat{t}}$, Harris Papapostolou ${ }^{a, c}$, Aline Machado de Castro ${ }^{d}$, Denise Maria Guimaraes Freire ${ }^{e}$, George J.E. Nychas ${ }^{a}$, Seraphim Papanikolaou ${ }^{a}$, Ioannis K. Kookos ${ }^{b, c, *}$, Apostolis Koutinas ${ }^{a, *}$

${ }^{\text {a }}$ Department of Food Science and Human Nutrition, Agricultural University of Athens, Iera Odos 75, Athens, Greece

${ }^{\mathrm{b}}$ Department of Chemical Engineering, University of Patras, Rio 26504, Patras, Greece

${ }^{\mathrm{c}}$ INVALOR: Research Infrastructure for Waste Valorization and Sustainable Management

${ }^{\mathrm{d}}$ Cenpes, Centro de Pesquisas Leopoldo Américo Miguez de Mello, Petrobras, Rio de Janeiro, Brazil

${ }^{\text {e }}$ Biochemistry Department, Chemistry Institute, Federal University of Rio de Janeiro, Cidade Universitária, Centro de Tecnologia, Bloco A, Lab 549, Rio de Janeiro, RJ, Brazil

$\$$ Equally contributed as first authors

* Corresponding authors: Ioannis K. Kookos (e-mail: i.kookos@chemeng.upatras.gr); Apostolis Koutinas (e-mail: akoutinas@aua.gr)

The number of pages, figures and tables are 13, 5 and 7 respectively. 

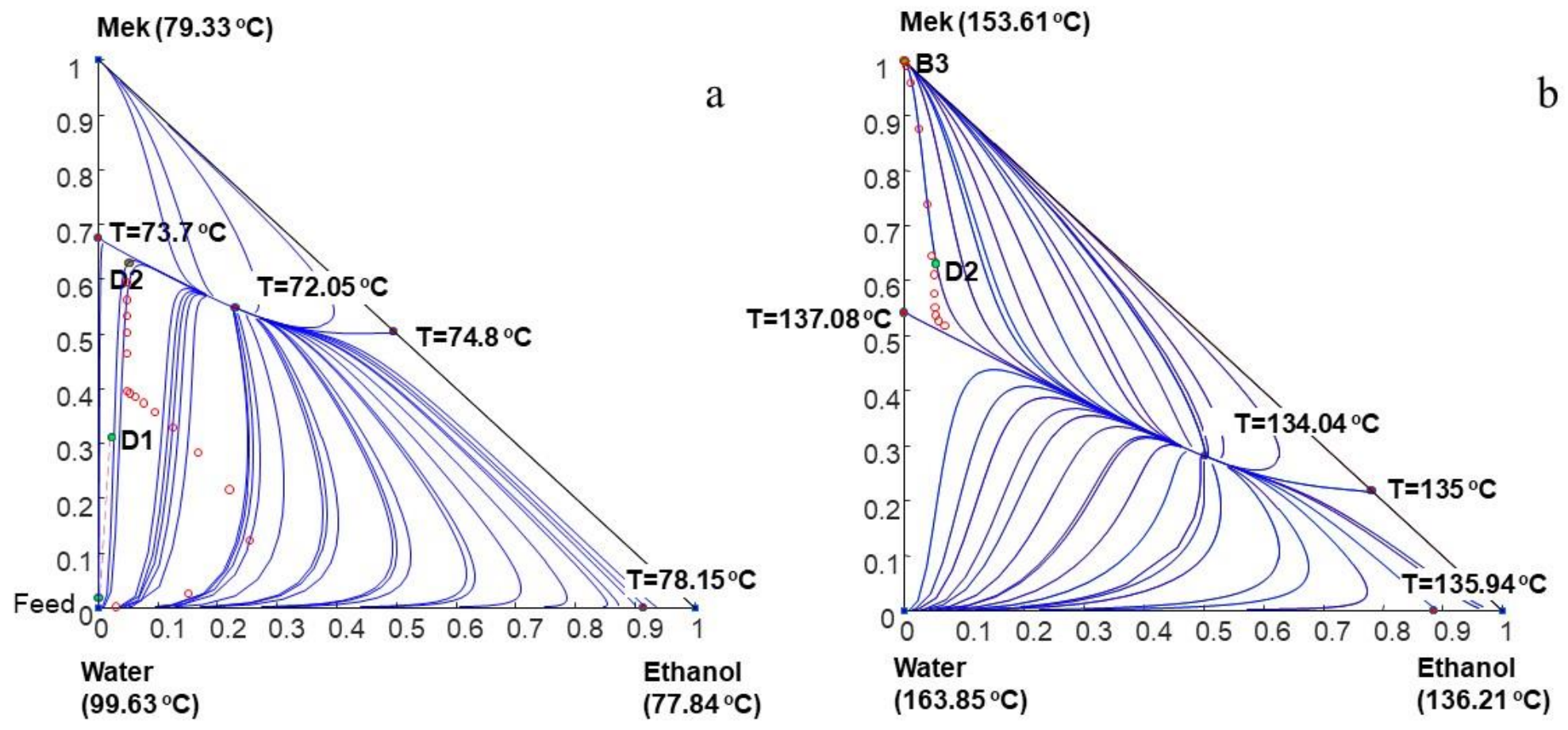

Figure S1. Residue curve map of the ternary system ethanol-MEK-water at 1 bar (a) and residue curve map of the ternary system ethanol-MEKwater at 6.8 bar (b). 

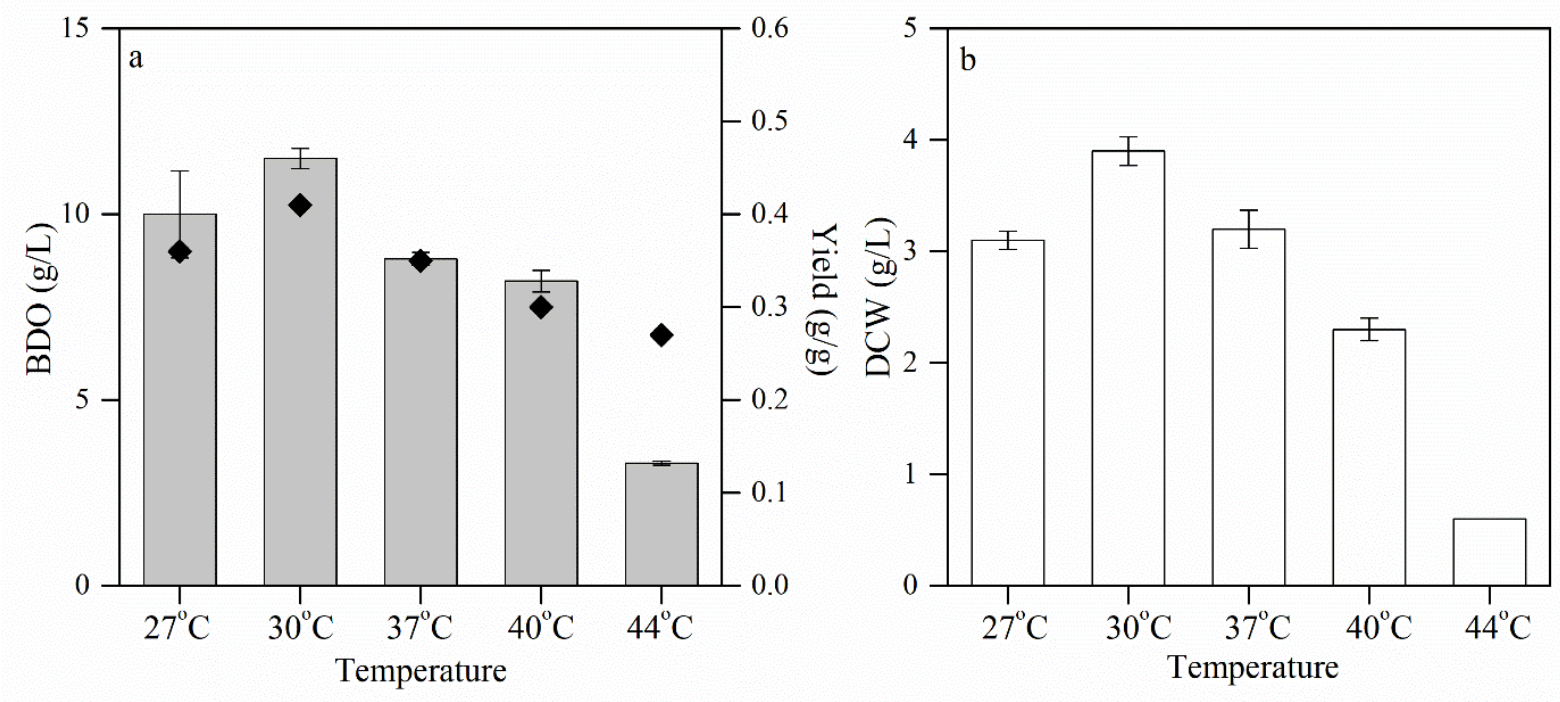

Figure S2. BDO production (bars), conversion yield ( $\bullet$ (a) and DCW (b) achieved in shake flask cultures under different cultivation temperatures 

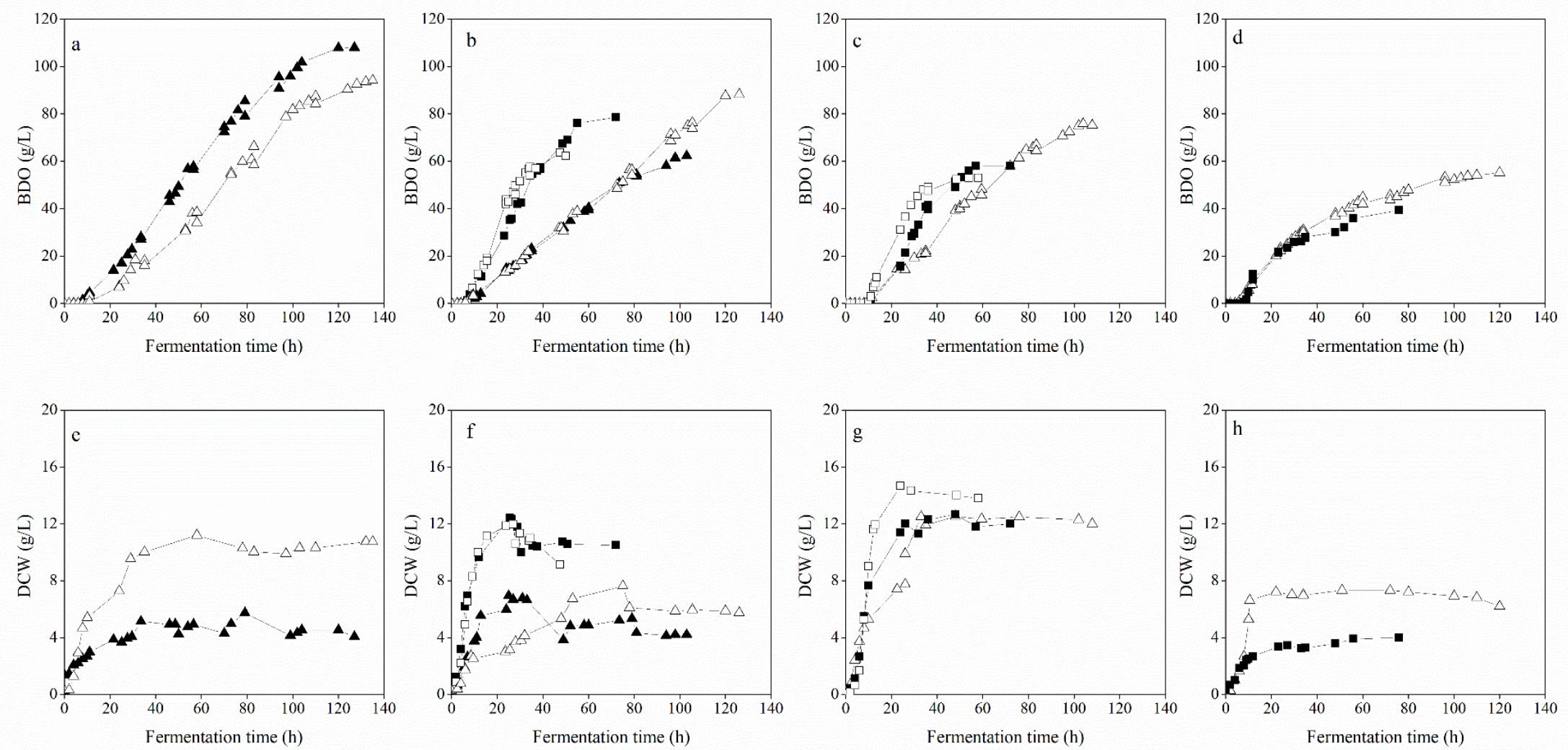

Figure S3 BDO and DCW during fed-batch fermentations using pure glycerol (a,e), crude glycerol 100-So (b,f), crude glycerol 90-So:10-Tf (c,g) and crude glycerol 65-So:35-Tf (d,h) at $k_{L} a$ values of 46-62 $\mathrm{h}^{-1}(\boldsymbol{\Delta}), 62 \mathrm{~h}^{-1}(\Delta), 77 \mathrm{~h}^{-1}(\mathbf{\square})$ and $83 \mathrm{~h}^{-1}(\square)$. 

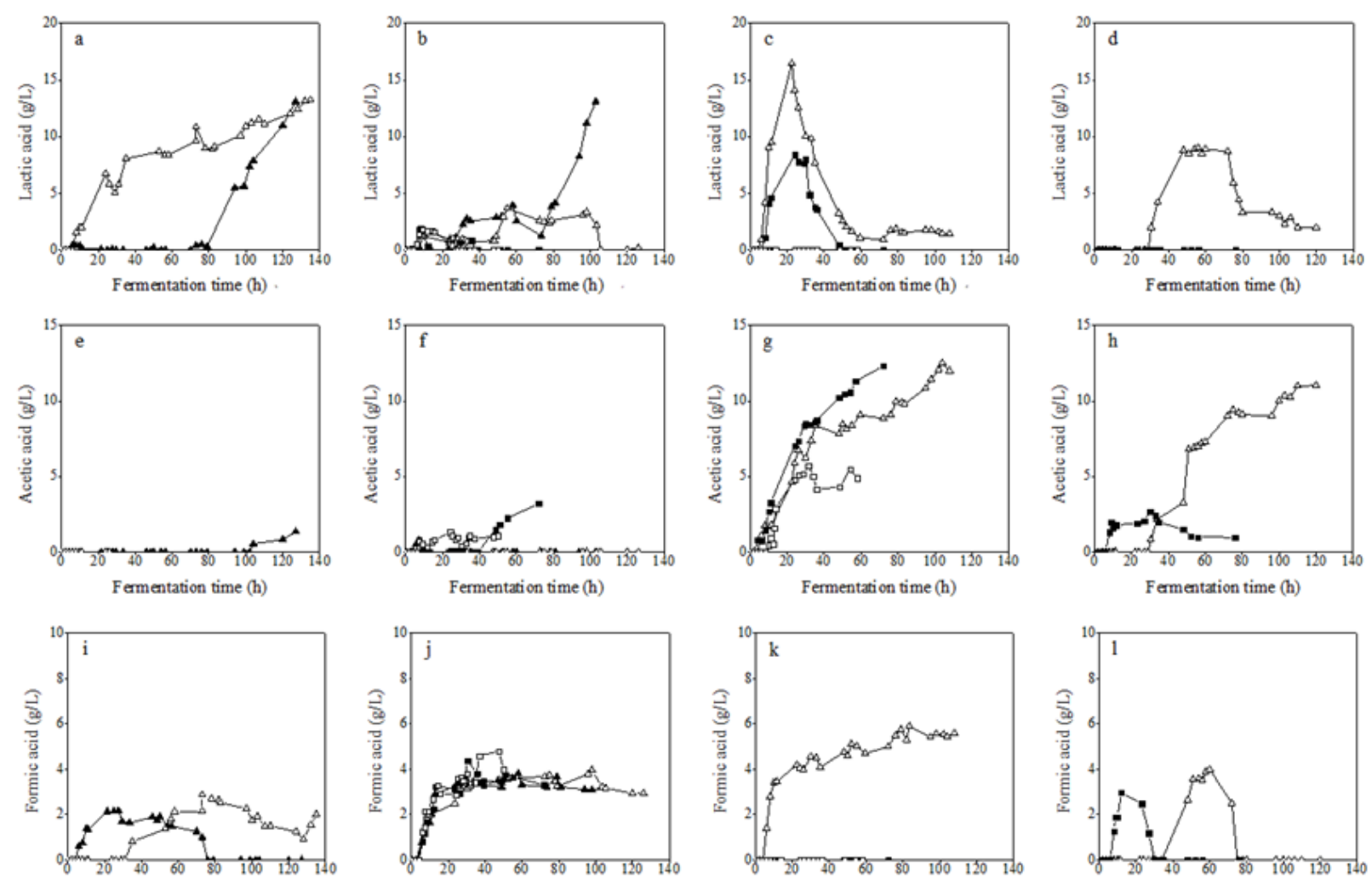

Fermentation time (h)

Fermentation time (h)

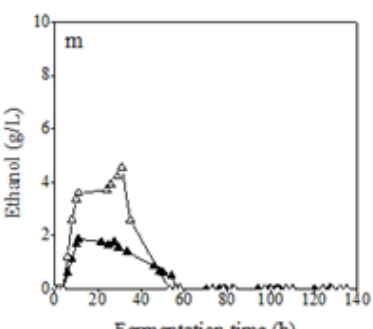

Fermentation time (h)
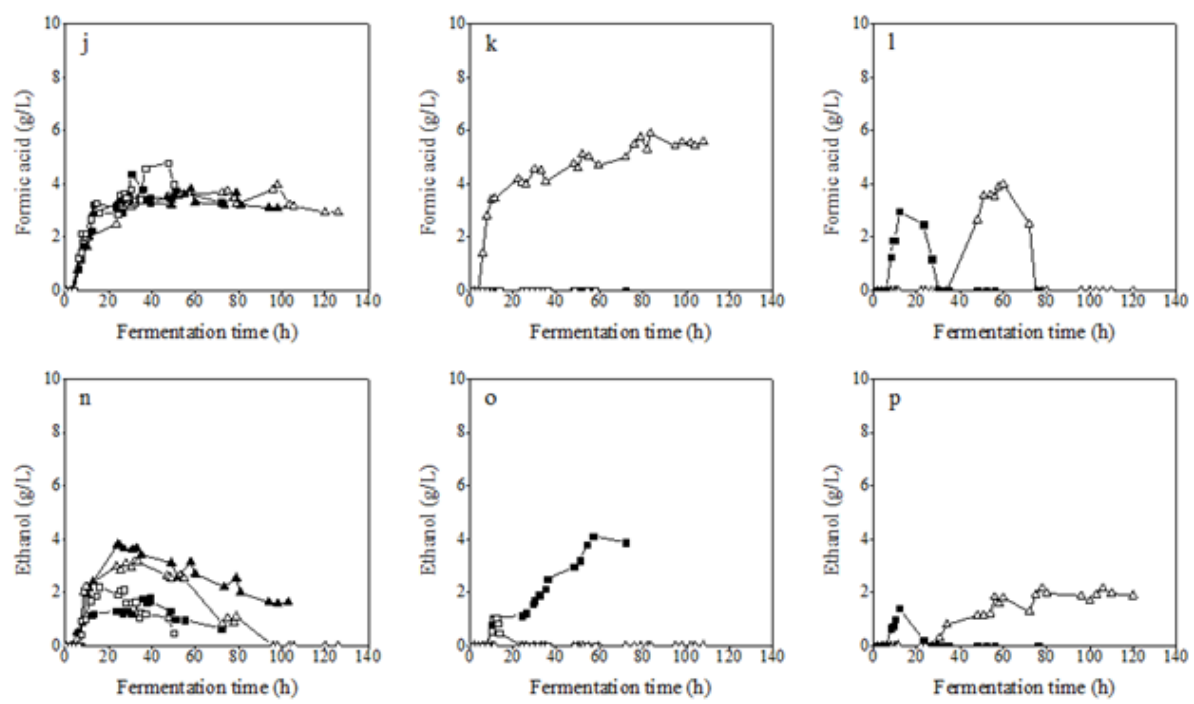

Figure S4 Organic acid and ethanol production using pure glycerol (a,e,i,m), crude glycerol 100-So (b,f,j,n), crude glycerol 90-So:10-Tf (c,g,k,o) and crude glycerol 65-So:35-Tf (d,h,1,p) at $k_{L} a$ values of 46-62 $\mathrm{h}^{-1}(\boldsymbol{\Delta}), 62 \mathrm{~h}^{-1}(\Delta), 77 \mathrm{~h}^{-1}(\mathbf{\square})$ and $83 \mathrm{~h}^{-1}(\square)$. 

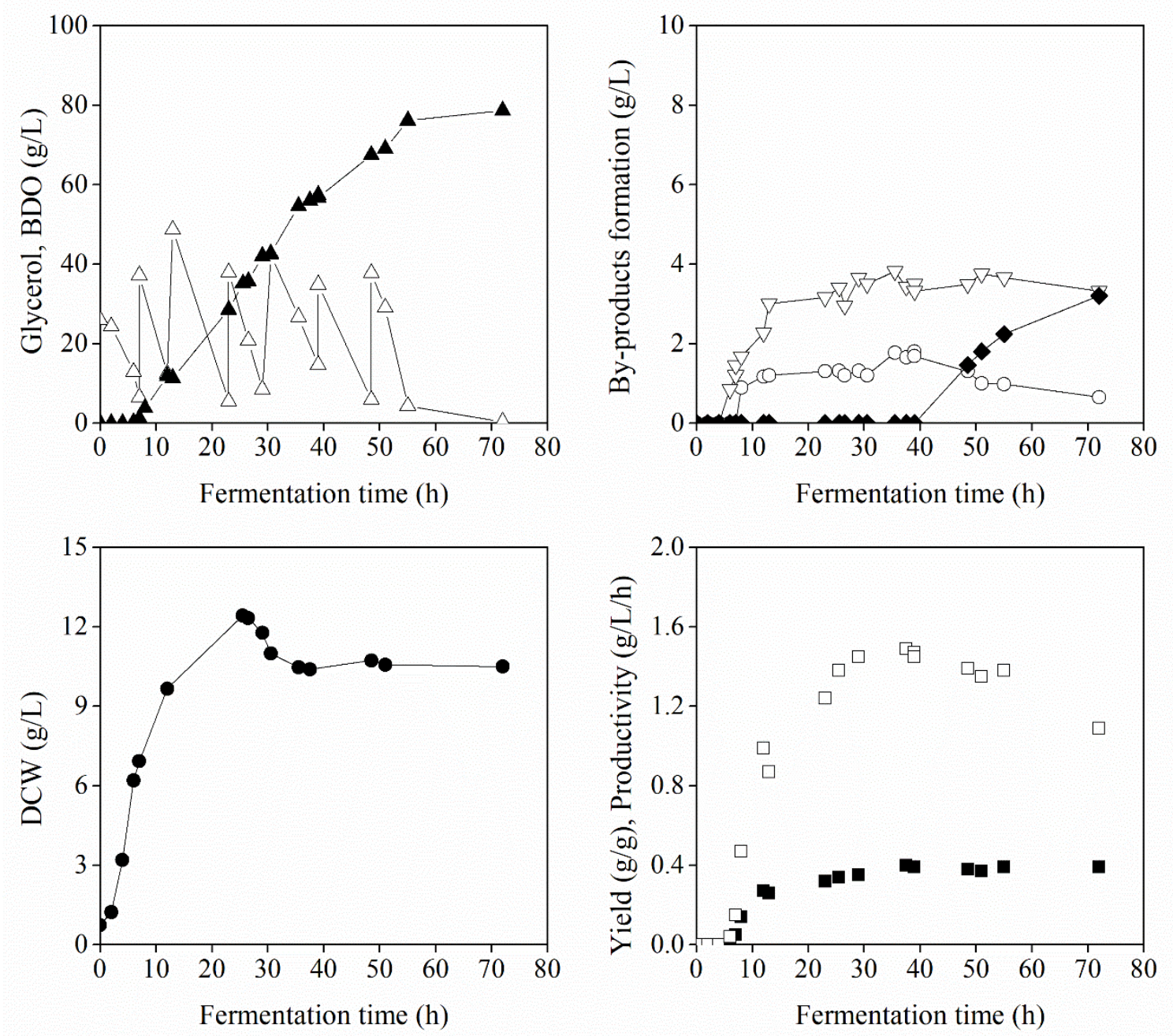

Figure S5. BDO $(\boldsymbol{\Delta})$, formic acid $(\nabla)$, acetic acid $(\bullet)$, ethanol $(\circ)$ and DCW $(\bullet)$ production as well as glycerol consumption $(\Delta)$, glycerol to BDO conversion yield ( $\mathbf{a})$ and productivity $(\square)$ during fed-batch fermentation using crude glycerol 100-So at $k_{L} a$ value of $77 \mathrm{~h}^{-1}$. 
Table S1 Optimal design parameters of Area 100 for the production of $10000 \mathrm{t}$ and $50000 \mathrm{t}$ $\mathrm{BDO} / \mathrm{y}$ from crude glycerol based on experimental results presented in this study as well as $50000 \mathrm{t} \mathrm{BDO} / \mathrm{y}$ from very high polarity cane sugar (VHPCS) as carbon source based on the fermentation efficiency reported by Maina et al. ${ }^{40}$

\begin{tabular}{ccc|c}
\hline $\begin{array}{c}\text { Process design and scheduling } \\
\text { parameters }\end{array}$ & \multicolumn{2}{|c|}{ Crude glycerol } & VHPCS \\
\cline { 2 - 4 } Number of bioreactors & $\mathbf{1 0 , 0 0 0 ~ t / y}$ & $\mathbf{5 0 , 0 0 0}$ t/y & $\mathbf{5 0 , 0 0 0 ~ t / y}$ \\
\hline Working bioreactor volume $\left(\mathrm{m}^{3}\right)$ & 5 & 11 & 6 \\
Total bioreactor volume $\left(\mathrm{m}^{3}\right)$ & 239.42 & 478.7 & 348.1 \\
Loading time (h) & 299.27 & 598.4 & 435.1 \\
Fermentation time $(\mathrm{h})$ & 4 & 4 & 2 \\
Uploading time (h) & 55 & 55 & 22 \\
Agitators per bioreactor & 15 & 6 & 5 \\
Total number of compressors \\
$\quad(8000$ SCFM/16 psig)
\end{tabular}


Table S2 Estimation of fixed capital investment for 50000 t/y BDO production via fermentation (Area 100) using very high polarity cane sugar as carbon source based on the fermentation efficiency reported by Maina et al. ${ }^{40}$

\begin{tabular}{|c|c|c|c|c|}
\hline $\begin{array}{c}\text { Unit } \\
\text { operation }\end{array}$ & Characteristic size & Data source & $\mathrm{C}_{p} \mathrm{M} \$$ @2017 & $\begin{array}{c}\text { Percentage of } \\
\text { total } C_{p}(\%)\end{array}$ \\
\hline $\mathrm{T}-101$ & $\mathrm{~V}=435.1 \mathrm{~m}^{3}, \mathrm{SS} 304$ & Humbird et al. ${ }^{30}$ & 0.319 & 4.00 \\
\hline E-101 & $\mathrm{A}=1000 \mathrm{~m}^{2}, \mathrm{SS} 316$ & Peters et al..$^{32}$ & 0.368 & 4.61 \\
\hline E-102 & $\mathrm{A}=58 \mathrm{~m}^{2}, \mathrm{SS} 316$ & Peters et al. ${ }^{32}$ & 0.041 & 0.51 \\
\hline E-103 & $\operatorname{Sch} 40, \mathrm{~d}=0.25, \mathrm{~L}=120 \mathrm{~m}$ & Peters et al. ${ }^{32}$ & 0.139 & 1.74 \\
\hline F-101-103 & $\begin{array}{c}34.8 \mathrm{~m}^{3}, 3.48 \mathrm{~m}^{3}, 0.35 \mathrm{~m}^{3} \\
\mathrm{SS} 304\end{array}$ & Humbird et al. ${ }^{30}$ & 0.726 & 9.10 \\
\hline F-104-109 & $6 \times 348.1 \mathrm{~m}^{3}, \mathrm{SS} 304$ & Humbird et al. ${ }^{30}$ & 2.881 & 36.09 \\
\hline A-104-109 & $6 \times 519$ hp, SS316 & Humbird et al. ${ }^{30}$ & 2.408 & 30.17 \\
\hline C-101-106 & $6 \times 306 \mathrm{~kW}, 16 \mathrm{psig}, \mathrm{CS}$ & Humbird et al. ${ }^{30}$ & 1.1 & 13.78 \\
\hline \multicolumn{2}{|c|}{ TOTAL (million \$) } & \multicolumn{3}{|c|}{7.982} \\
\hline \multicolumn{2}{|r|}{ FCI (million \$) } & \multicolumn{3}{|c|}{$\begin{array}{c}\approx 5 \times 7.982= \\
39.91\end{array}$} \\
\hline
\end{tabular}


Table S3 Estimation of fixed capital investment for MEK production (Area 200) via aqueous BDO conversion when very high polarity cane sugar is used as carbon source in the fermentation stage based on the fermentation efficiency reported by Maina et al. ${ }^{40}$

\begin{tabular}{|c|c|c|c|c|c|c|}
\hline \multirow{2}{*}{ UNIT } & \multirow{2}{*}{$\begin{array}{l}\text { Characteristic size } \\
\text { for } 50,000 \text { t/y BDO } \\
\text { production capacity }\end{array}$} & \multirow{2}{*}{ Data source } & \multicolumn{2}{|c|}{$\mathrm{C}_{p} M \$ \$ 2017$} & \multicolumn{2}{|c|}{$\begin{array}{c}\text { Percentage of total } C_{p} \\
(\%)\end{array}$} \\
\hline & & & $10,000 \mathrm{t} / \mathrm{y}$ & $50,000 \mathrm{t} / \mathrm{y}$ & $10,000 t / y$ & $50,000 \mathrm{t} / \mathrm{y}$ \\
\hline CF-201 & $\mathrm{Q}=69.61 \mathrm{~m}^{3} / \mathrm{h}$ & Green and Perry & 0.264 & 0.655 & 24.14 & 23.66 \\
\hline $\mathrm{R}-201$ & $\mathrm{~V}=90 \mathrm{~m}^{3} \mathrm{CS}$ & \multirow{3}{*}{ Turton et al. ${ }^{29}$} & 0.085 & 0.333 & 7.73 & 12.03 \\
\hline $\mathrm{R}-202$ & $\mathrm{~V}=90 \mathrm{~m}^{3} \mathrm{CS}$ & & 0.085 & 0.333 & 7.73 & 12.03 \\
\hline $\mathrm{T}-201$ & $\begin{array}{c}\mathrm{D}=3.53 \mathrm{~m}, \mathrm{H}=7.32 \mathrm{~m}, \\
11 \text { trays } \mathrm{SS} 316\end{array}$ & & 0.178 & 0.453 & 16.31 & 16.37 \\
\hline T-202 & $\begin{array}{c}\mathrm{D}=2.5 \mathrm{~m}, \mathrm{H}=13.9 \mathrm{~m} \\
20 \text { trays } \mathrm{SS} 316\end{array}$ & \multirow[t]{5}{*}{ Guthrie $^{a}$} & 0.201 & 0.465 & 18.38 & 16.80 \\
\hline $\mathrm{T}-203$ & $\begin{array}{c}\mathrm{D}=1.01 \mathrm{~m}, \mathrm{H}=10.24 \\
\mathrm{~m}, 15 \text { trays } \mathrm{SS} 316\end{array}$ & & 0.072 & 0.146 & 6.60 & 5.27 \\
\hline E-201 & $\mathrm{A}=56.22 \mathrm{~m}^{2} \mathrm{SS} 316$ & & 0.024 & 0.041 & 2.20 & 1.48 \\
\hline E-202 & $A=30.03 \mathrm{~m}^{2} \mathrm{SS} 316$ & & 0.024 & 0.031 & 2.17 & 1.12 \\
\hline E-203 & $A=59.33 \mathrm{~m}^{2} \mathrm{SS} 316$ & & 0.024 & 0.042 & 2.23 & 1.52 \\
\hline E-204 & $\mathrm{A}=171.54 \mathrm{~m}^{2} \mathrm{SS} 316$ & \multirow{5}{*}{ Peters et al. ${ }^{32}$} & 0.033 & 0.078 & 3.00 & 2.82 \\
\hline E-205 & $\mathrm{A}=29.13 \mathrm{~m}^{2} \mathrm{SS} 316$ & & 0.024 & 0.031 & 2.17 & 1.12 \\
\hline E-206 & $A=81.72 \mathrm{~m}^{2} \mathrm{SS} 316$ & & 0.026 & 0.049 & 2.38 & 1.77 \\
\hline E-207 & $\mathrm{A}=140.24 \mathrm{~m}^{2} \mathrm{SS} 316$ & & 0.031 & 0.08 & 2.80 & 2.89 \\
\hline E-208 & $A=29.27 \mathrm{~m}^{2} \mathrm{SS} 316$ & & 0.024 & 0.031 & 2.17 & 1.12 \\
\hline \multicolumn{2}{|c|}{ TOTAL (million \$) } & & 1.093 & 2.768 & & \\
\hline \multicolumn{2}{|c|}{ FCI (million \$) } & & $\begin{array}{c}\approx 5 \times \\
1.093= \\
5.47\end{array}$ & $\begin{array}{c}\approx 5 \times \\
2.768= \\
13.84\end{array}$ & & \\
\hline
\end{tabular}

${ }^{\mathrm{a}}$ Guthrie is the well-known methodology presented by Guthrie in the early $70 \mathrm{~s}$ 
Table S4 Raw materials cost $\left(\mathrm{C}_{\mathrm{RM}}\right)$ in Area 100 when crude glycerol is used as carbon source considering 50000 t/year BDO production capacity (excluding the cost of crude glycerol)

\begin{tabular}{ccccc}
\hline $\begin{array}{c}\text { Raw } \\
\text { material }\end{array}$ & $\begin{array}{c}\text { Concentration } \\
\left(\mathbf{k g} / \mathbf{m}^{\mathbf{3}}\right)\end{array}$ & $\begin{array}{c}\text { Annual } \\
\text { requirements } \\
(\mathbf{t} / \mathbf{y})\end{array}$ & $\begin{array}{c}\text { Unitary cost } \\
(\mathbf{\$} / \mathbf{k g})\end{array}$ & $\begin{array}{c}\text { Annual cost } \\
(\mathbf{M} \$ \mathbf{y})\end{array}$ \\
\hline$\left(\mathrm{NH}_{4}\right)_{2} \mathrm{SO}_{4}$ & 7.2 & $4,731.84$ & 0.15 & 0.710 \\
$\mathrm{MgSO}_{4} .7 \mathrm{H}_{2} \mathrm{O}$ & 0.3 & 197.16 & 0.42 & 0.083 \\
$\mathrm{CaCl}_{2} .7 \mathrm{H}_{2} \mathrm{O}$ & 0.09 & 59.15 & 0.15 & 0.009 \\
$\mathrm{MnSO}_{4} .7 \mathrm{H}_{2} \mathrm{O}$ & 0.0038 & 2.50 & 0.60 & 0.001 \\
$\left(\mathrm{NH}_{4}\right)_{2} \mathrm{HPO}_{4}$ & 6 & $3,943.20$ & 0.50 & 1.972 \\
$\mathrm{KOH}$ & 0.45 & 295.74 & 0.70 & 0.207 \\
$\mathrm{EDTA}$ & 0.51 & 335.17 & 2.00 & 0.670 \\
$\mathrm{FeSO}_{4} .7 \mathrm{H}_{2} \mathrm{O}$ & 0.0255 & 16.76 & 0.08 & 0.001 \\
$\mathrm{ZnSO}_{4} .7 \mathrm{H}_{2} \mathrm{O}$ & 0.0075 & 4.93 & 0.50 & 0.002 \\
\hline-0.0 & & & & $\mathrm{C}_{\mathrm{RM}}=3.655 \mathrm{M} \$ / \mathrm{y}$ \\
\hline
\end{tabular}


Table S5 Raw materials cost $\left(\mathrm{C}_{\mathrm{RM}}\right)$ in Area 100 when very high polarity cane sugar is used as carbon source (excluding the cost of very high polarity cane sugar) based on the fermentation efficiency reported by Maina et al. ${ }^{40}$ considering 50000 t/year BDO production capacity

\begin{tabular}{|c|c|c|c|c|}
\hline $\begin{array}{c}\text { Raw } \\
\text { material }\end{array}$ & $\begin{array}{c}\text { Concentration } \\
\left(\mathbf{k g} / \mathbf{m}^{3}\right)\end{array}$ & $\begin{array}{c}\text { Annual } \\
\text { requirements } \\
(t / y)\end{array}$ & $\begin{array}{c}\text { Unitary cost } \\
(\$ / \mathbf{k g})\end{array}$ & $\begin{array}{c}\text { Annual } \\
\text { cost } \\
(\mathrm{M} \$ / \mathbf{y})\end{array}$ \\
\hline$\left(\mathrm{NH}_{4}\right)_{2} \mathrm{SO}_{4}$ & 7.2 & $4,147.47$ & 0.15 & 0.622 \\
\hline $\mathrm{MgSO}_{4} .7 \mathrm{H}_{2} \mathrm{O}$ & 0.3 & 172.81 & 0.42 & 0.073 \\
\hline $\mathrm{CaCl}_{2} \cdot 7 \mathrm{H}_{2} \mathrm{O}$ & 0.09 & 51.84 & 0.15 & 0.008 \\
\hline $\mathrm{MnSO}_{4} .7 \mathrm{H}_{2} \mathrm{O}$ & 0.0038 & 2.19 & 0.6 & 0.001 \\
\hline$\left(\mathrm{NH}_{4}\right)_{2} \mathrm{HPO}_{4}$ & 6 & $3,456.23$ & 0.5 & 1.728 \\
\hline $\mathrm{KOH}$ & 0.45 & 259.22 & 0.7 & 0.182 \\
\hline EDTA & 0.51 & 293.78 & 2 & 0.588 \\
\hline $\mathrm{FeSO}_{4} .7 \mathrm{H}_{2} \mathrm{O}$ & 0.0225 & 12.96 & 0.08 & 0.001 \\
\hline $\mathrm{ZnSO}_{4} .7 \mathrm{H}_{2} \mathrm{O}$ & 0.0075 & 4.32 & 0.5 & 0.002 \\
\hline \multicolumn{4}{|c|}{ Total } & 3.205 \\
\hline
\end{tabular}


Table S6 Utilities cost (CUT) estimation in Area 100 and Area 200 when crude glycerol is used as carbon source considering 50000 t/year BDO production capacity

\begin{tabular}{|c|c|c|c|c|}
\hline UNIT & $\begin{array}{l}\text { Electricity } \\
\left(1^{6} \mathrm{kWh}\right)\end{array}$ & $\begin{array}{c}\text { Low pressure } \\
\text { steam } \\
\left(1^{6} t / y\right)\end{array}$ & $\begin{array}{c}\text { High pressure } \\
\text { steam } \\
\left(1^{6} \mathrm{t} / \mathrm{y}\right)\end{array}$ & $\begin{array}{l}\text { Cooling } \\
\text { water } \\
\left(1^{6} \mathbf{t} / \mathbf{y}\right)\end{array}$ \\
\hline \multicolumn{5}{|c|}{ Area $100-B D O$ production via fermentation } \\
\hline A-105-115 & 40.16 & - & - & - \\
\hline C-101-111 & 34.39 & - & - & - \\
\hline E-102 & - & 0.013 & - & - \\
\hline TOTAL & 74.55 & 0.013 & - & - \\
\hline Unit price & $0.0674 \$ / \mathrm{kWh}$ & $9.45 \$ / \mathrm{t}$ & $9.61 \$ / t$ & $0.0157 \$ / \mathrm{t}$ \\
\hline $\mathrm{M} \$ / \mathrm{y}$ & 5.025 & 0.123 & - & - \\
\hline \multicolumn{5}{|c|}{$\mathrm{C}_{\mathrm{UT}}=5.148 \mathrm{M} \$ / \mathrm{y}$} \\
\hline \multicolumn{5}{|c|}{ Area 200 - MEK production via aqueous BDO dehydration } \\
\hline CF-201 & 0.774 & - & - & - \\
\hline R-201-202 & 0.346 & - & - & 0.568 \\
\hline E-201 & - & - & 0.186 & - \\
\hline E-202 & - & - & - & 2.624 \\
\hline E-203 & - & 0.040 & - & - \\
\hline E-204 & - & - & - & 4.736 \\
\hline E-205 & - & 0.092 & - & - \\
\hline E-206 & - & - & - & 4.898 \\
\hline E-207 & - & 0.061 & - & - \\
\hline E-208 & - & - & - & 2.455 \\
\hline TOTAL & 1.12 & 0.193 & 0.186 & 15.281 \\
\hline Unit price & $0.0674 \$ / \mathrm{kWh}$ & $9.45 \$ / \mathrm{t}$ & $9.61 \$ / t$ & $0.0157 \$ / \mathrm{t}$ \\
\hline $\mathrm{M} \$ / \mathrm{y}$ & 0.075 & 1.824 & 1.787 & 0.240 \\
\hline $\mathrm{C}_{\mathrm{UT}}=3.926$ & & & & \\
\hline
\end{tabular}


Table S7 Utilities cost (CUT) estimation in Area 100 and Area 200 when very high polarity cane sugar is used as carbon source based on the fermentation efficiency reported by Maina et al. ${ }^{40}$ considering 50000 t/year BDO production capacity

\begin{tabular}{|c|c|c|c|c|}
\hline UNIT & $\begin{array}{l}\text { Electricity } \\
\left(10^{6} \mathrm{kWh}\right)\end{array}$ & $\begin{array}{c}\text { Low pressure } \\
\text { steam } \\
\left(1^{6} t / y\right)\end{array}$ & $\begin{array}{c}\text { Hign pressure } \\
\text { steam } \\
\left(1^{6} \mathrm{t} / \mathrm{y}\right)\end{array}$ & $\begin{array}{c}\text { Cooling } \\
\text { water } \\
\left(1^{6} \mathbf{t} / \mathbf{y}\right)\end{array}$ \\
\hline \multicolumn{5}{|c|}{ Area 100: BDO production from sucrose } \\
\hline A-104-109 & 14.08 & - & - & - \\
\hline C-101-106 & 11.13 & - & - & - \\
\hline E-102 & - & 0.012 & - & - \\
\hline TOTAL & 25.21 & 0.012 & - & - \\
\hline Unit price & $0.0674 \$ / \mathrm{kWh}$ & $9.45 \$ / \mathrm{t}$ & $9.61 \$ / \mathrm{t}$ & $0.0157 \$ / \mathrm{t}$ \\
\hline $\mathrm{M} \$ / \mathrm{y}$ & 1.699 & 0.113 & - & - \\
\hline \multicolumn{5}{|c|}{$\mathrm{C}_{\mathrm{UT}}=1.812 \mathrm{M} \$ / \mathrm{y}$} \\
\hline \multicolumn{5}{|c|}{ Area 200: MEK production from sucrose } \\
\hline $\mathrm{CF}-201$ & 0.774 & - & - & - \\
\hline R-201/202 & 0.298 & - & - & 0.380 \\
\hline E-201 & - & - & 0.174 & - \\
\hline E-202 & - & - & - & 1.569 \\
\hline E-203 & - & 0.051 & - & - \\
\hline E-204 & - & - & - & 3.220 \\
\hline E-205 & - & 0.04 & - & - \\
\hline E-206 & - & - & - & 1.528 \\
\hline E-207 & - & 0.054 & - & - \\
\hline E-208 & - & - & - & 1.423 \\
\hline TOTAL & 1.072 & 0.145 & 0.174 & 8.120 \\
\hline Unit price & $0.0674 \$ / \mathrm{kWh}$ & $9.45 \$ / t$ & $9.61 \$ / t$ & $0.0157 \$ / \mathrm{t}$ \\
\hline $\mathrm{M} \$ / \mathrm{y}$ & 0.072 & 1.370 & 1.672 & 0.127 \\
\hline $\mathrm{C}_{\mathrm{UT}}=3.241$ & $\$ y^{-1}$ & & & \\
\hline
\end{tabular}

